\title{
Atomic data from the IRON Project
}

\section{Electron excitation rates among the $3 \mathrm{~d}^{2}$ fine-structure levels of Ca-like Fe VII}

\author{
K.A. Berrington ${ }^{1}$, S. Nakazaki ${ }^{2}$, and P.H. Norrington ${ }^{3}$ \\ 1 School of Science \& Mathematics, Sheffield Hallam University, Sheffield S1 1WB, UK \\ 2 Department of Materials Science, Faculty of Engineering, Miyazaki University, Miyazaki 889-21, Japan \\ 3 Department of Applied Mathematics \& Theoretical Physics, Queen's University, Belfast BT7 1NN, UK
}

Received July 30; accepted October 22, 1999

\begin{abstract}
Electron excitation collision strengths of $3 \mathrm{~d}^{2}$ fine-structure transitions in Ca-like Fe VII are calculated using an R-matrix method, which included the 80 terms arising from the $3 \mathrm{~d}^{2}, 3 \mathrm{~d} 4 \mathrm{~s}, 3 \mathrm{~d} 4 \mathrm{p}, 3 \mathrm{~d} 4 \mathrm{~d}, 3 \mathrm{~d} 4 \mathrm{f}$ and $3 \mathrm{p}^{5} 3 \mathrm{~d}^{3}$ configurations. Extensive autoionizing resonance structures together with channel coupling is therefore explicitly included; relativistic effects are accounted for by a term-coupling transformation. A thermal average is taken to obtain the effective fine-structure collision strength as a function of electron temperature $T$, for $\log T(\mathrm{~K})=4.3-6$. Open $3 \mathrm{p}$-shell resonances are seen to considerably enhance the background collision strength, by several factors for some of the $3 \mathrm{~d}_{j \rightarrow j^{\prime}}^{2}$ transitions.
\end{abstract}

Key words: atomic data

\section{Introduction}

The present calculation is part of an international collaboration known as the IRON Project (Hummer et al. 1993, referred to as Paper I) to obtain accurate collision rates for fine-structure transitions. Here we calculate fine-structure collision strengths for transitions within the $3 \mathrm{~d}^{2}$ complex of Fe VII over a sufficiently wide and fine energy mesh, in order to be able to integrate over a Maxwellian distribution to obtain the effective collision strength, from which the excitation and de-excitation rate coefficients can easily be obtained (Paper I).

Earlier work on these collision strengths was by Norrington \& Grant (1987) using the Dirac R-matrix program. This is normally the most accurate approach when the scattering electron energy is comparable to the target level splitting. However, their calculation did not contain resonances arising from excited states above the $3 \mathrm{~d}^{2}$ states, and such resonance structures affect the collison strength right down to the $3 \mathrm{~d}^{2}$ states. A controversial feature of their results is that in the impact energy range from the highest $3 \mathrm{~d}^{2}$ threshold to beyond 3 Rydbergs, the collision strength from the Dirac R-matrix model decreased monotonically with energy, in contrast to that from the distorted-wave (DW) model of Nussbaumer \& Storey (1982) which increased. This difference was ascribed to channel coupling included the R-matrix method but not in DW; it is such differences we want to clarify.

\section{The model}

The basic atomic theory, the approximations and the computer codes employed in the IRON Project are described in Paper I of the A\&A series (Hummer et al. 1993). 80 terms are included in the target expansion, arising from the $3 \mathrm{~d}^{2}, 3 \mathrm{~d} 4 \mathrm{~s}, 3 \mathrm{~d} 4 \mathrm{p}, 3 \mathrm{~d} 4 \mathrm{~d}, 3 \mathrm{~d} 4 \mathrm{f}$ and $3 \mathrm{p}^{5} 3 \mathrm{~d}^{3}$ configurations. Figure 1 shows the calculated energy level diagram as an illustration of our model.

The radial orbitals for the Ca-like Fe ion target were taken from Clementi \& Roetti (1974) for $1 \mathrm{~s}, 2 \mathrm{~s}, 2 \mathrm{p}, 3 \mathrm{~s}, 3 \mathrm{p}, 3 \mathrm{~d}$. Also included were $n=4$ orbitals, optimised using Hibbert's (1975) variational program CIV 3 in the following way: 4 s optimised on the $3 \mathrm{~d} 4 \mathrm{~s}^{3,1} \mathrm{D}^{\mathrm{e}}$; $4 \mathrm{p}$ optimised on the $3 \mathrm{~d}_{4} \mathrm{p}^{3,1} \mathrm{D}^{\circ} ; 4 \mathrm{~d}$ optimised on the $3 \mathrm{~d} 4 \mathrm{~d}^{3,1} \mathrm{~F}^{\mathrm{e}}$; 4 f optimised on the $3 \mathrm{~d} 4 \mathrm{f}^{3,1} \mathrm{G}^{\mathrm{o}}$. The exponents of the $n=4$ orbitals are summarised in Table 1 .

A configuration interaction wavefunction is used to describe the target terms. The following configurations are included in the target description: $3 \mathrm{~d}^{2}, 3 \mathrm{~d} 41,414 \mathrm{l}^{\prime}$, $3 \mathrm{p}^{5} 3 \mathrm{~d}^{3}, 3 \mathrm{p}^{5} 3 \mathrm{~d}^{2} 4 \mathrm{~s}, 3 \mathrm{p}^{5} 3 \mathrm{~d} 4 \mathrm{~s}^{2}, 3 \mathrm{p}^{4} 3 \mathrm{~d}^{4}, 3 \mathrm{p}^{4} 3 \mathrm{~d}^{3} 4 \mathrm{~s}, 3 \mathrm{p}^{4} 3 \mathrm{~d}^{3} 4 \mathrm{p}$ and $3 \mathrm{~s} 3 \mathrm{p}^{6} 3 \mathrm{~d}^{3}$. This includes configurations to improve correlation in the target energies; similar configurations are included in the scattering " $N+1$ electron" system.

In order to assess our model target energies, we calculated level energies using the Breit-Pauli Hamiltonian with 


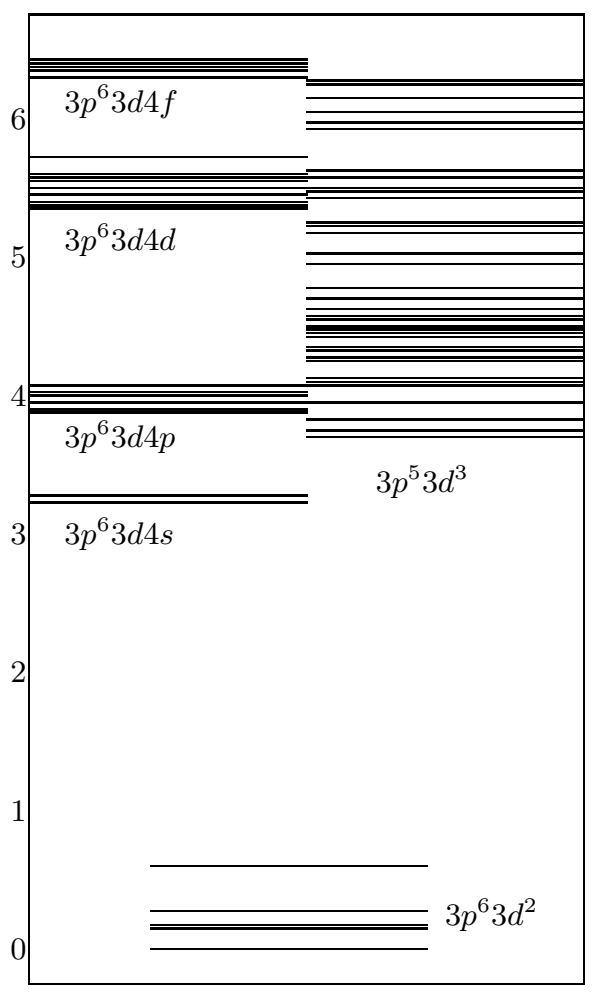

Fig. 1. The model atom: calculated energies (Ryds) of the 80 terms included for Fe VII

Table 1. Ca-like Fe target $n=4$ Slater-type orbital exponents for each power of $r$; the orbital coefficients are fixed by orthonormality conditions

\begin{tabular}{lcccc}
\hline$P_{\mathrm{nl}}$ & $r^{1}$ & $r^{2}$ & $r^{3}$ & $r^{4}$ \\
\hline $4 \mathrm{~s}$ & 3.05595 & 9.14246 & 1.28822 & 1.48174 \\
$4 \mathrm{p}$ & & 11.15581 & 4.58536 & 2.46411 \\
$4 \mathrm{~d}$ & & & 5.16564 & 1.97463 \\
4f & & & & 1.89000 \\
\hline
\end{tabular}

our wavefunction. These energies are shown in Table 2, where they are compared with those from the multiconfigurational Dirac-Fock (MCDF) calculation including full transverse Breit and QED contributions (Norrington \& Grant 1987) and with experiment (Ekberg 1981). Also included in Table 2 are energies which we calculated from our orbitals in a "frozen core" model (i.e. with no open pshell configuration interaction), which agrees remarkably well with the "MCDF" results (which was also a "frozen core" model), indicating that core correlation is more significant than relativistic effects and is responsible for most of the improvement of our present energies relative to experiment. In general, our calculated energy levels are now reasonably accurate for a scattering calculation, though we note that the only level which fails to improve in any calculation is $3 \mathrm{~d}^{2}{ }^{1} \mathrm{G}$.

The present 80-term calculation is in LS coupling, using the R-matrix programs of Berrington et al. (1995). The
Table 2. $3 \mathrm{~d}^{2}$ energy levels (Ryd) of Fe VII. The present calculation (together with a frozen-18-electron approximation) is compared with the multiconfigurational Dirac-Fock (MCDF) calculation of Norrington \& Grant (1987) and with experiment (Ekberg 1981)

\begin{tabular}{cccccc}
\hline$i$ & $3 \mathrm{~d}^{2}$ term & MCDF & frozen core & Present & Experiment \\
\hline & & & & & \\
1 & ${ }^{3} \mathrm{~F}_{2}$ & 0.0 & 0.0 & 0.0 & 0.0 \\
2 & ${ }^{3} \mathrm{~F}_{3}$ & 0.009 & 0.010 & 0.011 & 0.010 \\
3 & ${ }^{3} \mathrm{~F}_{4}$ & 0.021 & 0.023 & 0.024 & 0.021 \\
4 & ${ }^{1} \mathrm{D}_{2}$ & 0.193 & 0.194 & 0.167 & 0.159 \\
5 & ${ }^{3} \mathrm{P}_{0}$ & 0.223 & 0.224 & 0.196 & 0.183 \\
6 & ${ }^{3} \mathrm{P}_{1}$ & 0.226 & 0.227 & 0.200 & 0.186 \\
7 & ${ }^{3} \mathrm{P}_{2}$ & 0.233 & 0.236 & 0.209 & 0.194 \\
8 & ${ }^{1} \mathrm{G}_{4}$ & 0.294 & 0.298 & 0.298 & 0.264 \\
9 & ${ }^{1} \mathrm{~S}_{0}$ & 0.715 & 0.711 & 0.634 & 0.611 \\
\hline
\end{tabular}
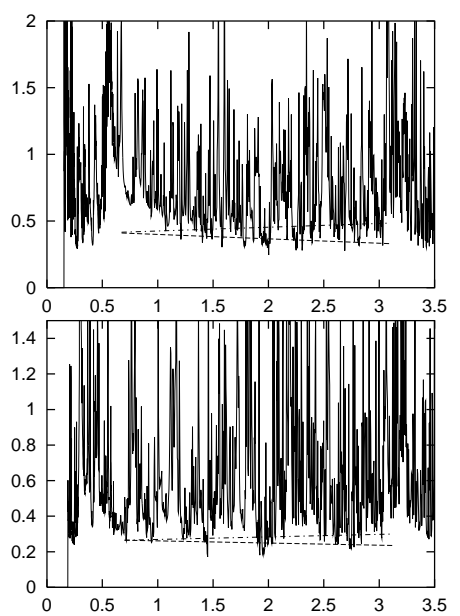

Fig. 2. Collision strength for electron excitation from the ground state ${ }^{3} \mathrm{~F}_{2}$ to ${ }^{1} \mathrm{D}_{2}$ (upper plot) and ${ }^{3} \mathrm{P}_{1}$ (lower) of $\mathrm{Fe}$ VII: - present 80-term R-matrix calculation; - - - 9-level Dirac R-matrix (Norrington \& Grant 1987); - - - - - - distorted-wave (Nussbaumer \& Storey 1982)

R-matrix boundary is at 9.6 a.u., we include 16 continuum terms per channel and mass correction and Darwin nonfinestructure relativistic terms. A transformation to intermediate coupling is applied to the T-matrix using the JAJOM procedure of Saraph (1978), with term coupling included amongst the $3 \mathrm{~d}^{2}$ terms (though the coupling coefficients are not very significant: the largest is between ${ }^{1} \mathrm{D}_{2}$ and ${ }^{3} \mathrm{P}_{2}$ where it is $5 \%$ ). This model is used in the resonance region between the $3 \mathrm{~d}^{2}$ states and the higher excited states to a maximum of 16 Ryds. A "top-up" in angular momentum is applied, and an explicit calculation to $J=20.5$ at the higher energies confirms convergence of these forbidden transitions.

\section{The results}

In Figs. 2 and 3 we compare calculated fine-structure collision strengths from the present 80 -term R-matrix plus 
Table 3. Effective collision strengths for Fe VII $3 \mathrm{~d}^{2}$ fine structure transitions $\left(i-i^{\prime}\right.$ using the indexation of Table 2$)$ as a function of $\log T . A=$ Calculated E2 and M1 transition probability of the upper state $\left(\mathrm{s}^{-1}\right.$, with a superscript indicating a power of ten factor)

\begin{tabular}{|c|c|c|c|c|c|c|c|c|c|}
\hline$i-i^{\prime}$ & $A$ & 4.3 & 4.5 & 4.75 & 5.0 & 5.25 & 5.5 & 5.75 & 6.0 \\
\hline $1-2$ & $.325^{-1}$ & 3.71 & 3.35 & 2.98 & 2.69 & 2.48 & 2.20 & 1.79 & 1.35 \\
\hline $1-3$ & $.167^{-8}$ & 1.30 & 1.17 & 1.02 & .926 & .862 & .776 & .634 & .469 \\
\hline $1-4$ & 3.25 & 1.01 & .959 & .918 & .873 & .813 & .721 & .593 & .455 \\
\hline $1-5$ & .135 & .295 & .299 & .299 & .302 & .305 & .292 & .259 & .217 \\
\hline $1-6$ & $.502^{-1}$ & .619 & .633 & .628 & .623 & .619 & .585 & .504 & .403 \\
\hline $1-7$ & .174 & .516 & .549 & .548 & .534 & .518 & .473 & .387 & .288 \\
\hline $1-8$ & $.959^{-3}$ & 1.14 & 1.24 & 1.26 & 1.20 & 1.11 & .971 & .786 & .595 \\
\hline $1-9$ & .181 & .134 & .115 & .097 & .087 & .080 & .071 & .057 & .043 \\
\hline $2-3$ & $.466^{-1}$ & 4.55 & 4.11 & 3.64 & 3.30 & 3.04 & 2.71 & 2.21 & 1.66 \\
\hline $2-4$ & .603 & 1.38 & 1.29 & 1.23 & 1.16 & 1.08 & .952 & .780 & .596 \\
\hline $2-5$ & & .228 & .235 & .231 & .226 & .222 & .207 & .174 & .133 \\
\hline $2-6$ & $.762^{-1}$ & .807 & .833 & .837 & .837 & .837 & .794 & .693 & .564 \\
\hline $2-7$ & $.697^{-1}$ & 1.02 & 1.06 & 1.06 & 1.05 & 1.03 & .964 & .818 & .637 \\
\hline $2-8$ & .343 & 1.59 & 1.74 & 1.76 & 1.68 & 1.56 & 1.36 & 1.11 & .837 \\
\hline $2-9$ & & .190 & .162 & .138 & .123 & .113 & .100 & .081 & .061 \\
\hline $3-4$ & $.139^{-2}$ & 1.72 & 1.60 & 1.51 & 1.42 & 1.32 & 1.16 & .943 & .716 \\
\hline $3-5$ & & .171 & .187 & .191 & .186 & .181 & .167 & .139 & .104 \\
\hline $3-6$ & & .649 & .690 & .692 & .677 & .663 & .616 & .516 & .390 \\
\hline $3-7$ & $.735^{-1}$ & 1.89 & 1.94 & 1.95 & 1.94 & 1.93 & 1.84 & 1.60 & 1.30 \\
\hline $3-8$ & .503 & 2.06 & 2.25 & 2.28 & 2.18 & 2.02 & 1.77 & 1.43 & 1.09 \\
\hline $3-9$ & & .249 & .213 & .181 & .162 & .149 & .132 & .107 & .080 \\
\hline $4-5$ & $.472^{-6}$ & .159 & .172 & .179 & .178 & .170 & .154 & .128 & .099 \\
\hline $4-6$ & $.572^{-1}$ & .490 & .531 & .553 & .544 & .515 & .462 & .382 & .292 \\
\hline $4-7$ & .191 & 1.022 & 1.06 & 1.07 & 1.03 & .952 & .833 & .668 & .495 \\
\hline $4-8$ & $.414^{-2}$ & 2.01 & 2.02 & 1.91 & 1.76 & 1.62 & 1.43 & 1.16 & .889 \\
\hline $4-9$ & 26.7 & .509 & .473 & .468 & .483 & .492 & .480 & .439 & .384 \\
\hline $5-6$ & $.115^{-2}$ & .350 & .370 & .380 & .380 & .389 & .402 & .375 & .306 \\
\hline $5-7$ & $.139^{-7}$ & .315 & .324 & .324 & .329 & .347 & .363 & .342 & .287 \\
\hline $5-8$ & & .155 & .164 & .178 & .186 & .183 & .166 & .138 & .106 \\
\hline $5-9$ & & .030 & .035 & .040 & .041 & .039 & .034 & .028 & .021 \\
\hline $6-7$ & $.743^{-2}$ & 1.13 & 1.17 & 1.18 & 1.20 & 1.25 & 1.31 & 1.23 & 1.03 \\
\hline $6-8$ & & .466 & .495 & .536 & .558 & .549 & .500 & .416 & .320 \\
\hline $6-9$ & 6.88 & .088 & .105 & .120 & .123 & .116 & .103 & .084 & .064 \\
\hline $7-8$ & $.454^{-4}$ & .857 & .903 & .961 & .988 & .965 & .875 & .726 & .558 \\
\hline $7-9$ & 1.11 & .173 & .197 & .220 & .225 & .215 & .192 & .159 & .122 \\
\hline $8-9$ & & .255 & .310 & .346 & .345 & .320 & .273 & .209 & .146 \\
\hline
\end{tabular}

JAJOM model and the 9-level Dirac R-matrix model (Norrington \& Grant 1987) in the scattering energy region up to the $3 \mathrm{~d} 4 \mathrm{~s}$ thresholds, and we also compare with distorted-wave calculations of Nussbaumer \& Storey (1982).

These figures illustrate for the first time the effect of resonances, not included in previous calculations, in the collision strength over this energy range. These resonances are converging to $3 \mathrm{~d} 41$ and $3 \mathrm{p}^{5} 3 \mathrm{~d}^{3}$ excited states, and mask any differences in the prediction of the two earlier calculations of a straight-line background, indicating instead a much more complex energy dependency.

The large low-lying resonance near 0.5 Ryd was identified by an analysis of the Hamiltonian eigenvalues and vectors in the vicinity, and is due to $3 \mathrm{p}^{5} 3 \mathrm{~d}^{3} 4 \mathrm{p} N+1$ electron configurations, which are seen to dominate the low energy collision rate particularly for ${ }^{3} \mathrm{~F}_{2}-{ }^{1} \mathrm{G}_{4}$. This confirms the importance of including $3 \mathrm{p}$-hole correlation in the scattering calculation for this ion.

Because of the importance of such near-threshold resonances, we repeated the calculation using experimental energies to correct our target energies, with no appreciable difference in the final rates.

As a further test, we used the Breit-Pauli R-matrix approach in the $3 \mathrm{~d}^{2}$ threshold energy region, thus including fine-structure splitting explicitly. Only 5 terms (i.e. 9 levels) were included in this test because of computational constraints, though the same $N+1$ electron configurations were included as in our main 80-term R-matrix plus JAJOM run. Replacing the low energy "JAJOM" collision strengths with those from the Breit-Pauli test run, and recalculating the effective collision strengths, gives 

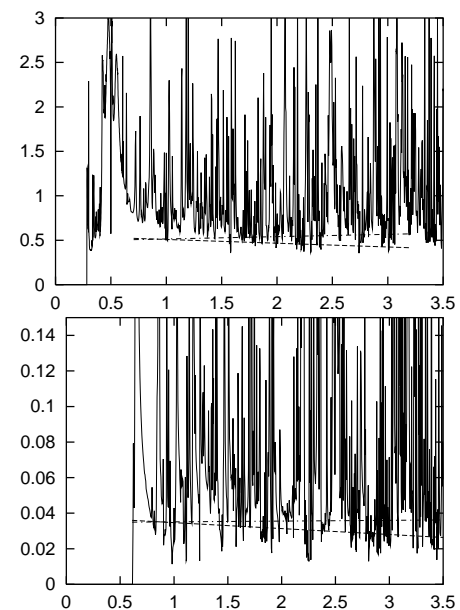

Fig. 3. Collision strength for electron excitation from the ground state ${ }^{3} \mathrm{~F}_{2}$ to ${ }^{1} \mathrm{G}_{4}$ (upper plot) and ${ }^{1} \mathrm{~S}_{0}$ (lower) of Fe VII: - present 80-term R-matrix calculation; - - - 9-level Dirac R-matrix (Norrington \& Grant 1987); - - - - distorted-wave (Nussbaumer \& Storey 1982)

an estimate of the error due to numerical difficulties in establishing the relative position of thresholds and resonances in the "JAJOM" results presented in Table 3 as $20 \%$.

Table 3 tabulates the resulting effective collision strength for Fe VII $3 \mathrm{~d}^{2}$ transitions in the electron temperature range $\log T(\mathrm{~K})=4.3-6$. This includes the temperature of maximum ionic abundance which is given by Shull \& Van Steenberg (1982) as $\log T=5.6$. We also calculated, using our wavefunctions in CIV3, the E2 and M1 transition probability of these forbidden transitions, and we show these also in Table 3.

In conclusion, we have used an 80-term R-matrix model to calculate collision strengths for $3 \mathrm{~d}^{2}$ transitions which show in some cases strong enhancement of background due to large and broad open-shell resonances, and therefore show big differences with earlier compilations based on the 9-level Dirac R-matrix approach (Keenan \& Norrington 1987, 1991). We have discussed the difficulties in modelling such cases, in a computer-tractable way, and estimate our results at $20 \%$ at best. Further improvements would require larger basis sets with more configuration interaction and relativistic effects to accurately represent the resonance structure near the thresholds.

Acknowledgements. This work was done with the support of a PPARC grant GR/K97608.

\section{References}

Berrington K.A., Eissner W., Norrington P.H., 1995, Comput. Phys. Commun. 92, 290

Clementi E., Roetti C., 1974, Atom. Data Nucl. Data Tab. 14, 177

Ekberg J.O. 1981, Phys. Scr. 23, 7

Hibbert A., 1975, Comput. Phys. Commun. 9, 141

Hummer D.G., Berrington K.A., Eissner W., Pradhan A.K., Saraph H.E., Tully J.A., 1993, A\&A 279, 298 (Paper I)

Keenan F.P., Norrington P.H., 1987, A\&A 181, 370

Keenan F.P., Norrington P.H., 1991, ApJ 368, 486

Norrington P.H., Grant I.P., 1987, J. Phys. B: At. Mol. Phys. 20,4869

Nussbaumer H., Storey P.J., 1982, A\&A 113, 21

Saraph H.E., 1978, Comp. Phys. Commun. 15, 247

Shull J.M., Van Steenberg M., 1982, ApJS 48, 95 